\title{
Current capabilities and challenges for optical coherence tomography as a high impact non-destructive imaging modality
}

Adrian Bradu, Manuel Marques, Sylvain Rivet, Adrian Podoleanu

Adrian Bradu, Manuel Marques, Sylvain Rivet, Adrian Podoleanu, "Current capabilities and challenges for optical coherence tomography as a high impact non-destructive imaging modality," Proc. SPIE 10831, Seventh International Conference on Lasers in Medicine, 108310E (10 August 2018); doi: $10.1117 / 12.2281502$

Event: Seventh International Conference on Lasers in Medicine, 2017, Timisoara, Romania 


\title{
Current Capabilities and Challenges for Optical Coherence Tomography as a High Impact Non-destructive Imaging Modality
}

\author{
Adrian Bradu*a, Manuel Marques ${ }^{\mathrm{a}}$, Sylvain Rivet ${ }^{\mathrm{b}}$ and Adrian Podoleanu ${ }^{\mathrm{a}}$ \\ ${ }^{a}$ Applied Optics Group, School of Physical Sciences, University of Kent, CT2 7NH Canterbury, \\ United Kingdom \\ ' Laboratoire d'Optique et de Magnetisme EA938, IBSAM, Univ. Bretagne Occidentale, C.S. 93837, \\ 29238 Brest Cedex 3, France \\ *a.bradu@kent.ac.uk
}

\begin{abstract}
Non-destructive sensing and imaging within the body of materials is essential for quality control and very importantly for the development of new materials, equally for industrial and medical applications. Conventional non-destructive testing (NDT) methods, such us ultrasound, exhibit low imaging resolutions, of hundreds of microns and typically require a direct contact between the probe and the sample to be investigated. The speed at which the standard NDT methods performs is also quite restricted. The development of optical coherence tomography (OCT) applications in the field of NDT have grown immensely over the past years, offering faster, higher resolution images in a completely contactless environment with the sample. Optical Coherence Tomography brings a plethora of benefits to the current non-destructive methods. However, a multitude of challenges still need to be overcome to truly make OCT the technique of choice for NDT applications. In this paper, a short overview of the main challenging of producing cross-sectional, transversal and volumetric OCT images are presented with an emphasize on OCT's capabilities and limitations in producing images in real-time. Real-time OCT images of various samples produced using the Master/Slave technique developed within the Applied Optics Group at the University of Kent are demonstrated.
\end{abstract}

Keywords: Optical coherence tomography, non-destructive testing

\section{CHALLENGES IN (NDT) OCT}

\subsection{Need for better lateral resolution}

The beauty of the OCT technology is that the axial (longitudinal) resolution in the image is decoupled from the transversal (lateral) resolution. While the axial resolution is determined by the bandwidth of the optical source employed, the transversal resolution depend on the interface optics in the sample arm of the interferometer, mainly on the numerical aperture of the microscope objective employed to focus light on the object, in the same way as in confocal microscopy. Optical sources with sufficient broad optical spectrum provide an excellent axial resolution. To improve the lateral resolution, a high numerical aperture microscope objective must be used. The drawback of this approach is a limited axial image restricted to the extension of the confocal gate. A solution suitable for high numerical aperture interface optics can be Gabor filtering [1]. Using this technique, data acquisition is repeated for several focus positions, corresponding to various shifts of the confocal gating profile through the sample. The images hence obtained are fused in a final larger image covering a large axial range. Such an approach was applied so far successfully, however as the data acquisition must be performed multiple times, the real time operation of the instrument is limited.

\subsection{Need for increased axial imaging range}

Both implementations of spectral (Fourier) domain OCT, respectively spectrometer based (Sp) and swept source (SS), can be used to produce images with high speed and high sensitivity. SS-OCT has some advantages over the Sp-OCT including a higher imaging speed, a longer axial range and a quite uniform sensitivity over the axial range. Although SSOCT technology seems to be the method of choice when a long axial range is required, there are still applications, here including the NDT ones that could benefit from an even more extended imaging range. Unfortunately, due to the finite coherence length of the lasers used, the axial imaging range is still limited. An exception from this makes the tunable vertical cavity surface emitting lasers (VCSEL) and akinetic light sources, which can provide a long axial range, 
exceeding centimeters. However, both VCSELs and akinetic light sources commercially available are costlier than the microelectromechanical system (MEMS) SSs widely available and in use in various settings.

Irrespective of the way in which OCT is implemented, SS (MEMS, VCSEL, akinetic) and Sp-OCT the generation of images is based on a Fourier transform (FT), therefore mirror terms reduce the achievable axial range by a factor of 2. An elegant solution to extend the axial range in SS-OCT is the use of the coherence revival exhibited by some commercially available external cavity tunable laser (ECTL) swept sources [2].

The coherence revival method employs the interference between the waves reflected from the interferometer arms that originate from waves that suffered different multiple reflections inside the swept laser cavity. This method can easily be implemented, as it only requires an alteration of the optical path difference (OPD) between the two arms of the interferometer. When using the coherence revival, there is no need for additional hardware, the axial resolution is not affected as the sweeping range is the same, while the sensitivity drop is negligible especially for NDT applications where there are practically no constrains on the optical power used to illuminate the sample.

Although, this method is interesting, as it is based on enhancing the current capabilities of existent hardware at no extra cost, it has some disadvantages, one of them being the fact that now intensive dispersion compensation is required, as twice the laser cavity length is within the total optical path difference creating the spectral modulation to be decoded. To compensate for the extra dispersion due to the laser cavity, only software solutions can be used which normally leads to heavy computation.

\subsection{Miniaturisation}

In some NDT applications, the OCT imaging instruments must be equipped with small, flexible probes hence easy access to areas of the investigated samples not easily reachable is possible. Therefore, the development of the endoscopic (EOCT) and handheld (HHOCT) able to produce high resolution long axial imaging range is very important.

Both, EOCT and HHOCT instruments can be devised in two ways: common path (where the reference arm of the interferometer is created by placing a partial reflector close to the sample to be imaged), and conventional non-common path, where the arms of the interferometer are independently controlled. The first way does not offer robustness as there is practically no way to actuate on the position of the partial reflector, especially in EOCT, and as a result the achievable axial imaging range is restricted. A non-common path configuration has also some limitations. First, as all EOCT and HHOCT instruments are built using optical fibers, matching their lengths in the arms of the interferometer with micrometer accuracy is challenging.

To compensate for the dispersion in the interferometer, both hardware, and numerical methods can be implemented. However, these methods are not perfect and lead to an increase in the complexity of the systems or to heavy computation. Second, data must be calibrated (re-sampled), process which cannot be perfectly performed and leads to a heavy computational load, which in the end impacts the real-time operation of the instrument.

\subsection{Real-time operation}

The production of real-time images in OCT is typically limited by the complex mathematical operations required to process data after acquisition. Traditionally, in order to produce a cross-sectional image, in both implementations of OCT, camera and swept source, each channelled spectrum acquired while scanning the probing beam over the sample is subject to a fast Fourier transform (FFT). Before the FFT, several preparatory signal processing steps are necessary, such as zero padding, spectral shaping, apodization, dispersion compensation and data resampling. Without these preparatory steps, axial resolution as well as sensitivity suffer [3]. Some of the preparatory operations are relatively time consuming. However, the main issue is that these steps can only be sequentially executed hence the production of the OCT images in real-time is limited. Several techniques involving both hardware [4] and various software [5] solutions have been demonstrated to successfully eliminate or diminish the execution time of the preparatory steps.

The most demanding preparatory steps in terms of computation time are data resampling and compensation for dispersion mismatch between the arms of the interferometer. To eliminate the resampling step, in camera based OCT, hardware solutions were proposed. However, this requires careful adjustment of the hardware components and the use of extra optical components in the interferometer introduces losses. In swept source OCT, the swept sources are often equipped with a supplementary k-clock [6] that adds to the cost of the source, consisting in an extra interferometer equipped with a photo-detector at least twice as fast as the highest frequency component to be detected in the main 
imaging interferometer from the maximum depth. It also requires a two input sophisticated digitizer. Even so, this does not yield a perfect resampling and limits the axial range of the images.

Other techniques such as using an additional light source that produces several spectral lines in the region of interest of the spectrometer, parametric iteration methods, phase linearization techniques, and automatic calibrations have been also proposed. All these methods are normally computationally expensive and limit the real-time operation of OCT systems.

Dispersion compensation is the other important limiting factor in obtaining high-resolution OCT images. Both hardware and software methods have been used by various research groups to overcome it.

Numerical dispersion compensation is more cost-effective and flexible in is done numerically (in software) as the hardware methods which involve a perfect matching of the dispersion between the arms of the interferometer is not feasible all the time. Various numerical techniques to compensate for dispersion such as auto-focusing have been developed. However, all the numerical algorithms used to compensate for dispersion involve Hilbert transformations, phase corrections, filtering, etc, hence a heavy computational loading and therefore numerical dispersion compensation has to be performed as a post-processing step.

Solutions involving parallel computing hardware enable huge increases in performance, taking advantage of devices such as graphics processing units (GPUs) [7] or field-programmable gate arrays (FPGAs) [8]. By significantly reducing the computation time of preparatory steps, true real-time production of B-scan images becomes possible. However, as some of the preliminary steps are often based on iterative calculations, errors in correctly preparing the data are unavoidable.

As the computational requirements for high-speed SD-OCT image processing usually exceed the capabilities of most computers, the display rates of OCT images rarely match the acquisition rates for most devices. After the preparatory steps, most image analysis and diagnosis become a post-processing operation. A true real-time display of processed cross-sectional and en-face, could vastly benefit NDT applications that require instant feedback of image information.

\section{SOLUTION: SIGNAL PROCESSING USING THE MASTER SLAVE APPROACH}

To tackle the drawbacks of the current OCT instruments presented above, we developed imaging instruments having at their core a technique developed in our group: Master/Slave (MS) $[9,10]$. These instruments can meet the demands of various NDT applications such as high lateral and axial resolution, long axial range and real-time operation. They are also offering flexibility as the MS approach allows for easy, quick adjustments of the instruments if required by a specific application.

The benefits of the MS method originate from the fact that the method does not involve any FFT in order to generate images [9]. As there is no FFT, there is no need for any of the preparatory steps mentioned in the previous section, and as the method is only based on multiplication of channelled spectra, it is highly parallelizable [11]. As a result, as no sequential mathematical operations are involved, the MS method can operate in true real time. The MS method compares, raw, unprocessed spectra which incorporate all the information about nonlinearities and unbalanced dispersion. As a result, there is no need for data resampling or procedures to compensate for unbalanced dispersion. An enhanced MS OCT instrument is by definition an instrument free of non-linearities and perfectly compensated for dispersion.

Various MS enhanced OCT imaging instruments were devised in our group and used for different NDT applications, from bench top, to handheld and endoscopic instruments, operating at various spectral ranges and producing very long axial ranges [12-16]. In Fig. 1, some hand-held developed in our group are presented. Depending on the need of the application, they were interfaced with optics ensuring a very long axial imaging range (Fig. 1A) or a high lateral resolution as for example the HH-OCT instrument shown in Fig. 1C. 


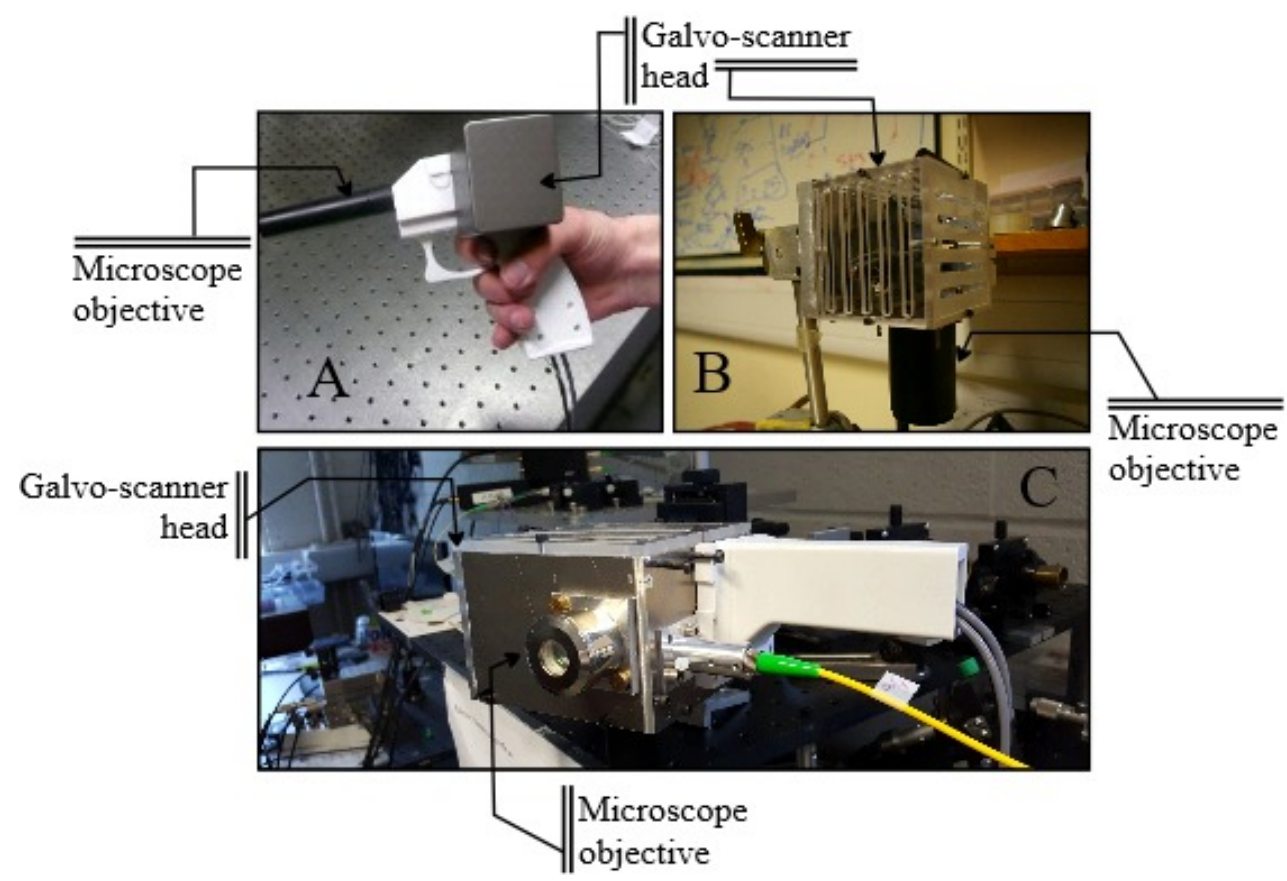

Figure 1. Examples of hand-held devices developed in the AOG suitable for NDT applications. Long axial range HHOCT instrument developed in the $1300 \mathrm{~nm}(\mathrm{~A}$ and $\mathrm{C}$ ) and $1050 \mathrm{~nm}(\mathrm{~B})$ spectral ranges. B.

Using the hand held device presented in Fig. 1(C), high resolution images similar to those presented in Fig. 2 can be produced. This instrument operates in the $1300 \mathrm{~nm}$ spectral range hence it provides an enhanced depth penetration. As, at the same time it is equipped with a high numerical aperture microscope objective, high lateral resolution images can be generated. As an example, in Fig. 2(A1) and 2(A2), high lateral resolution cross-sectional images of the human nail and of the interface skin/nail are presented. In Fig. 2(A3), a high lateral resolution en-face image of a medicinal caplet is shown.

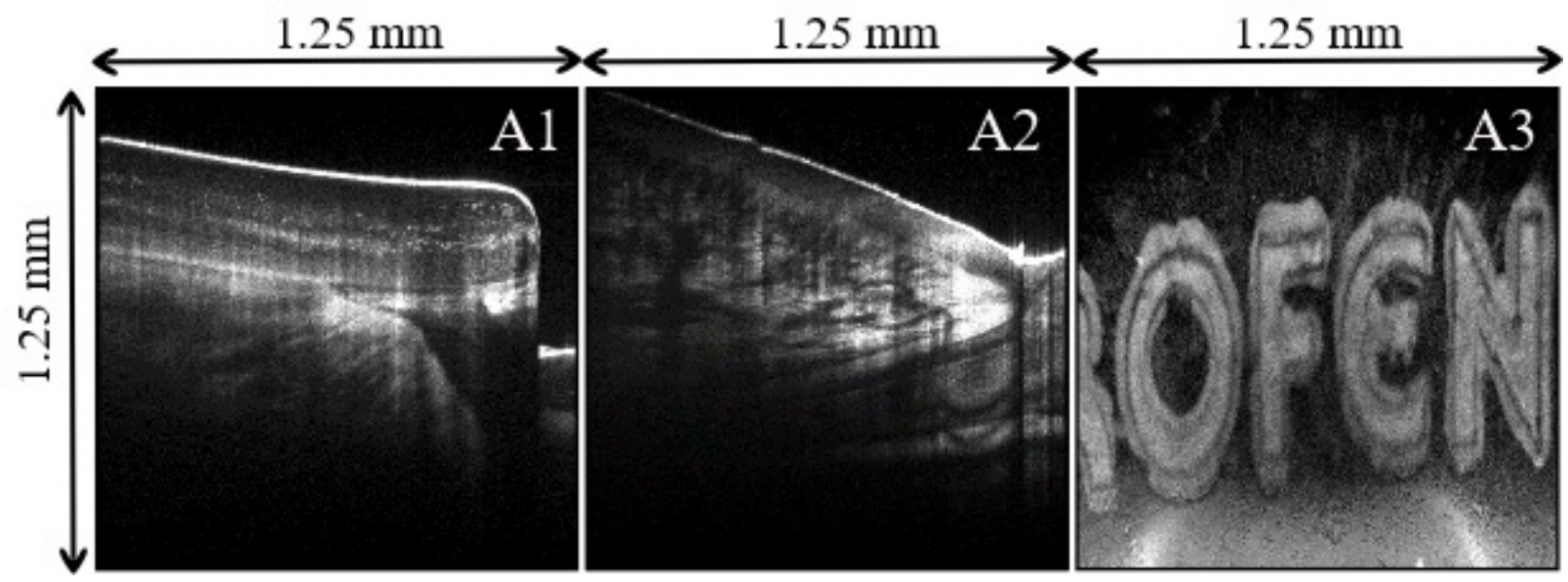

Figure 2. High-resolution cross-sectional images of the human nail (A1), junction nail/skin (A2) and an en-face view of a pill. The images were obtained using the HH-OCT instrument shown in Fig. 1C.

All the instruments we developed so far having at their core the MS technology can be used for a variety of NDT applications. As an illustrative application, we have imaged various pills. The images shown in Fig. 3 were also produced using the hand-held instrument presented in Fig. 1(C) equipped with a tunable laser whose sweeping speed is $100 \mathrm{kHz}$. As these images have 500 lateral pixels, data to produce each cross-section image (Fig. 3(A1-A2)) was acquired in $5 \mathrm{~ms}(200 \mathrm{~Hz})$. As for these particular samples there is no need for an axial imaging range longer than about $2 \mathrm{~mm}$, the number of axial points in each image could be reduced in such a way that each pixel in the image covers in 
depth a length equal to the optical axial resolution. With a limited number of points to be computed axially, the master/slave approach could produce the cross-sectional images in real-time (within $5 \mathrm{~ms}$ ). In conventional, FFT based OCT, this approach is not possible as the number of axial points generated by the FFT operation is fixed, always equal the half of the number of sampling points each channelled spectrum is sampled into. Each en-face image shown in Fig. 3 (B1-B4) has a size of $500 \times 500$ pixels was produced in real-time at a frame rate of $0.4 \mathrm{~Hz}$.

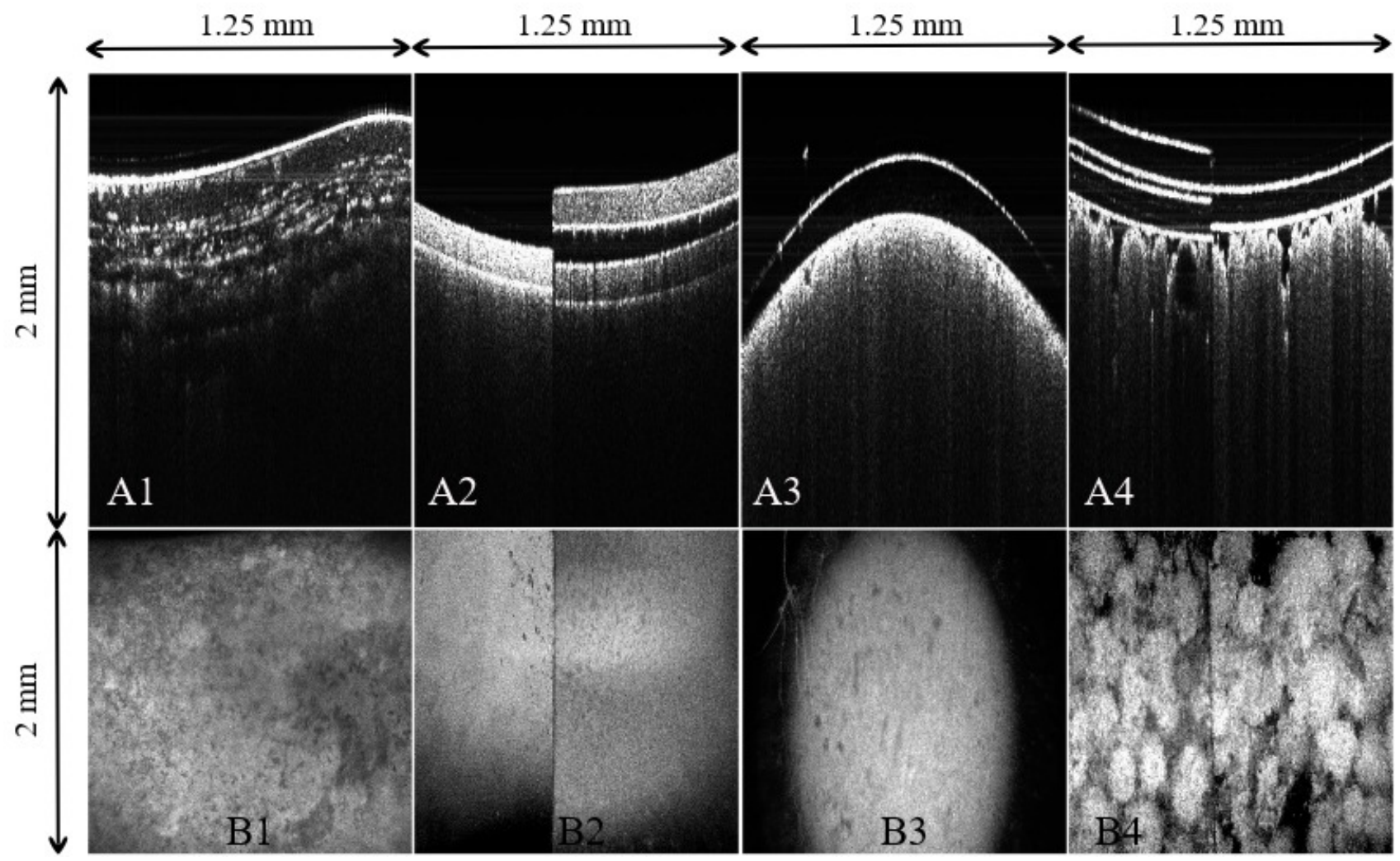

Figure 3. Top row: cross-sectional images of various caplets (A1 and A3) and medicinal capsules (A2 an A4). Bottom row: en-face images of the corresponding caplets and medicinal capsules presented in the top row. In A2 and A4 as well in B2 and B4, the junction between the two halves of the capsules is clearly visible.

\section{CONCLUSIONS}

In this manuscripts a short review of some drawbacks of the current OCT technology was presented. To overcome these limitations, the use of the Master/Slave approach was proposed and some illustrative images produced using MS enhanced OCT instruments developed in our group suitable to be used for NDT applications were demonstrated. The Master/Slave approach has several advantages over the conventional FFT based OCT technology, such as no need for pre-processed data and perfectly balanced dispersion in the interferometer. These advantages make the MS enhanced OCT instruments excellent candidates for NDT applications where extra flexibility of the instruments is needed in terms of lateral resolution and axial imaging range.

In terms of real-time production of the images, current CPUs are capable of data processing as required by the Master/Slave approach, with no need to resort to FPGA or GPUs. However, the MS potential is there, where the numerous comparison operations needed can be performed on FPGAs and GPUs, to improve even further the real-time processing. As an MS enhanced OCT instrument is capable of producing cross-sectional, en-face and volumetric images in real-time, such an instrument is very appropriate to be used in an industrial setting where swift imaging of the samples is required as for example for fast quality control along a production line. 


\section{ACKNOWLEDGEMENTS}

The authors would like to thank: EPSRC grant REBOT (EP/N019229/1/), ERC-POC ERC ADASMART 754695, MarieCurie, Intra-European Fellowship for Career Development 625509, UBAPHODESA as well as NIHR Biomedical Research Centre at Moorfields Eye Hospital NHS Foundation Trust and the UCL Institute of Ophthalmology and the Royal Society Wolfson research merit award.

\section{REFERENCES}

[1] Rolland, J. P., Meemon, P., Murali, S., Thompson, K. P., and Lee, K., "Gabor-based fusion technique for Optical Coherence Microscopy," Optics Express 18, 3632-3642 (2010).

[2] Dhalla, A-H., Nankivil, D., and Izatt, J., "Complex conjugate resolved heterodyne swept source optical coherence tomography using coherence revival," Biomed. Opt. Express 3, 633-649 (2012).

[3] Leitgeb, R., Drexler, W., Unterhuber, A., Hermann, B., Bajraszewski, T., Le, T., Stingl, A., and Fercher, A., "Ultrahigh resolution Fourier domain optical coherence tomography," Opt. Express 12, 2156-2165 (2004).

[4] $\mathrm{Hu}, \mathrm{Z}$., and Rollins, A.M., "Fourier domain optical coherence tomography with a linear-in-wavenumber spectrometer," Opt. Lett. 32, 3525-3527 (2007).

[5] Zhang, K., and Kang, J.U., "Graphics processing unit accelerated non-uniform fast Fourier transform for ultrahigh-speed, real-time Fourier-domain OCT," Opt. Express 18, 23472-23487 (2010).

[6] Liu, B., Azimi, E., and Brezinski, M.E., "True logarithmic amplification of frequency clock in SS-OCT for calibration," Biomed. Opt. Express 2, 1769-1777 (2011).

[7] Zhang, K., and Kang, J.U., "Real-time 4D signal processing and visualization using graphics processing unit on a regular nonlinear-k Fourier-domain OCT system," Opt. Express 18, 11772-11784 (2010).

[8] Ustun, T.E., Iftimia, N.V., Ferguson, R.D. and Hammer, D.X., "Real-time processing for Fourier domain optical coherence tomography using a field programmable gate array," The Rev. Scientific Instrum. 79, 114301 (2008).

[9] Podoleanu, A., and Bradu, A., "Master-slave interferometry for parallel spectral domain interferometry sensing and versatile 3D optical coherence tomography," Opt. Express 21, 19324-19338 (2013).

[10] Rivet, S., Maria, M., Bradu, A., Feuchter, T., Leick, L., and Podoleanu, A., "Complex master slave interferometry," Opt. Express 24, 2885-2904 (2016).

[11] Bradu, A., Kapinchev, K., Barnes, F., and Podoleanu, A., "On the possibility of producing true real-time retinal cross-sectional images using a graphics processing unit enhanced master-slave optical coherence tomography system,” J. Biomed. Opt., 20, 076008 (2015).

[12] Cernat, R., Bradu, A., Israelsen, N., Bang, O., Rivet, S., Keane, P., Heath, D-G., Rajendram, R., and Podoleanu, A., "Gabor fusion master slave optical coherence tomography," Biomed. Opt. Express 8, 813-827 (2017).

[13] Sinescu, C., Bradu, A., Duma, V., Topala, F., Negrutiu, M., and Podoleanu, A., "Effects of Temperature Variations during Sintering of Metal Ceramic Tooth Prostheses Investigated Non-Destructively with Optical Coherence Tomography," Appl. Sci. 7, 552 (2017).

[14]Bradu, A., Rivet, S., Podoleanu, A., "Master/slave interferometry - ideal tool for coherence revival swept source optical coherence tomography," Biomed. Opt. Express 7, 2453-2468 (2016).

[15] Sinescu, C., Bradu, A., Duma, V., Topala, F., Negrutiu, M., and Podoleanu, A., "Temperature variations in sintering ovens for metal ceramic dental prostheses. Non-destructive assessment using OCT," Proc. SPIE 10473, Lasers in Dentistry XXIV, 104730C (14 February 2018).

[16] Marques, M.J., Rivet, S., Bradu, A., and Podoleanu, A., "Novel software package to facilitate operation of any spectral (Fourier) OCT system," Proceedings Volume 10416, Optical Coherence Imaging Techniques and Imaging in Scattering Media II; 104160B (1 August 2017). 